\title{
MÓDULO DE GESTÃO DE CONTEÚDO (CONCEITOS) BASEADO EM GAMIFICAÇÃO, ATRELADO AO SISTEMA DE AVALIAÇÃO DA APRENDIZAGEM (SAA)
}

ITU/SP JUNHO/2018

\author{
Dilermando Piva Junior - Fatec / CPS - pivajr@gmail.com \\ Bruno Marthe de Almeida - Fatec-Itu - brunomarthe@hotmail.com \\ Tipo: Investigação Científica (IC) \\ Natureza: Planejamento de Pesquisa \\ Categoria: Métodos e Tecnologias \\ Setor Educacional: EDUCAÇÃO SUPERIOR
}

\begin{abstract}
RESUMO
Indicadores educacionais e pesquisas recentes vem mostrando que a utilização de metodologias ativas ao longo do processo educacional resulta em um ganho significativo da aprendizagem, e, consequentemente, maior atratividade e redução de índices de evasão em cursos superiores de graduação. Em recém pesquisa junto aos estudantes que utilizam o Sistema de Avaliação de Aprendizagem (SAA), observou-se uma melhor ligação entre conteúdo e processos de avaliação formativa se faziam necessários. Com vistas a potencializar a utilização do sistema SAA pelos estudantes e a possibilidade de implementação de metodologias ativas pelos docentes que utilizam 0 SAA, como por exemplo, gamificação, o desenvolvimento de um módulo de gerenciamento de conteúdo, com forte ligação com o processo de avaliação do SAA se faz necessário. Assim, este projeto tem como objetivo desenvolver um módulo de gestão de conteúdo, baseado em gamificação, atrelado ao sistema SAA. Uma vez desenvolvido esse novo módulo, o mesmo deverá ser testado em casos reais, junto aos estudantes, com a coleta de informações que indiquem se a implementação conseguiu potencializar a utilização do sistema pelos estudantes e melhorar o processo de aprendizagem.
\end{abstract}

Palavras-chave: Gamificação, Avaliação Formativa, SAA. 


\section{Introdução}

É possível observar que a prática de avaliação nas Instituições de Ensino Superior está, muitas vezes, centrada no emprego de avaliações somativas como forma básica de "medir" quanto conteúdo foi "aprendido" pelos estudantes (Moraes, 2014). As razões são diversas e não cabe aqui discuti-las, é certo que a legislação tem contribuído para consolidar esse procedimento, especialmente em cursos desenvolvidos a distância, dado que é exigida avaliação presencial que prepondere sobre as demais formas avaliativas e, com isso se dê o avanço para etapas posteriores do desenvolvimento de um dado curso.

No mundo contemporâneo, a mudança do paradigma educacional do ensino para a aprendizagem tem permitido novas possibilidades de condução dos processos avaliativos, que passam a ter foco no processo, no dia-a-dia, no "feedback" dado aos estudantes para seu desenvolvimento e, não apenas por uma questão de escala, o emprego da tecnologia tem se tornado cada vez mais importante e necessário.

É nesse contexto que se insere o Sistema de Avaliação da Aprendizagem - SAA(Piva Jr et al, 2016), que é uma ferramenta que potencializa a ação docente, tornando possível a aplicação de avaliações formativas ao longo do processo de ensino-aprendizagem, independentemente da metodologia utilizada (presencial ou a distância), de haver ou não a inversão da sala de aula, ou de serem utilizados ou não ambientes de aprendizagem. Uma das principais vantagens de proporcionar a verificação da aprendizagem por meio de métodos formativos consiste na possibilidade de prover correções de rumo - ou retomada dos mesmos, caso seja verificado um desvio prejudicial aos objetivos do projeto e ao aprendizado dos estudantes, de forma geral.

Com a utilização do sistema SAA, após um planejamento inicial, o professor consegue estabelecer um acompanhamento mais individualizado, mesmo trabalhando com um número grande de estudantes.

A Figura 1(A), a seguir, representa a sequência de planejamento de uma determinada disciplina que deve ser realizada por um docente no SAA. Depois de realizados os cadastros iniciais, deve-se observar as etapas operacionais frequentes do sistema. Essas etapas são descritas na Figura 1(B). Em termos práticos, uma vez que se chega a uma data planejada de aula, o sistema aponta que pode ser realizado o lançamento das presenças dos estudantes. 


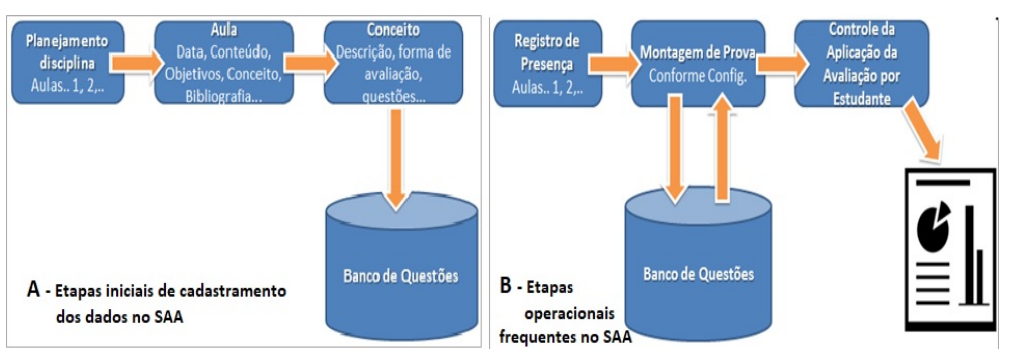

Figura 1 - Etapas realizadas no SAA

Uma vez pontadas as presenças, apenas e tão somente para os alunos que estiveram presentes àquela atividade, o sistema irá montar uma avaliação (individualizada) que será associada aos estudantes que estiveram presentes. Uma mensagem por e-mail será enviada a cada estudante presente para que ele se lembre de que existe uma avaliação a ser realizada, com informações da disciplina, da aula e a quantidade de minutos que o estudante deve reservar (em média) para respondê-la. Após a realização da avaliação, o sistema disponibilizará uma série de relatórios e gráficos para que o professor, o tutor, o estudante, o coordenador e o diretor da unidade, possam tomar as ações convenientes

O SAA foi desenvolvido e aplicado junto a disciplinas de cursos na área de Computação e Informática, em modelos tradicionais de curso (cursos presenciais). Os testes foram realizados em três Faculdades de Tecnologia do Centro Paula Souza. A primeira etapa envolveu duas turmas de um mesmo professor, num total de 109 estudantes. A segunda etapa, cinco professores em sete turmas/disciplinas diferentes, num total de 387 estudantes. Na primeira etapa, aferiu-se um ganho médio de aprendizagem da ordem de $17,64 \%$ e, na segunda etapa, os resultados mostraram valores com melhoria da ordem de $20 \%$.

Ao longo desses testes, o SAA foi avaliado pelos professores e estudantes. Os resultados dessa avaliação indicaram pontos fortes e fracos da aplicação do sistema, e do próprio sistema. Um dos pontos indicados para melhoria é a forma como conteúdo e avaliação são vinculados. Segundo as opiniões dos estudantes, deve existir uma ligação mais forte entre o conteúdo e a avaliação, para que os pontos que careçam de melhor compreensão possam ser acessados diretamente pelos estudantes, após a realização das avaliações. Além dessa ligação, é fundamental que o processo de avaliação formativa, garanta que o estudante tenha passado por determinados conteúdos antes de avaliá-los. O simples rastreamento das ações dos estudantes ao longo das interações com os conteúdos pode garantir isso, entretanto, a literatura mostra que não são suficientes. A junção da avaliação formativa com metodologias ativas é o que resulta em indicadores mais relevantes e significativos no processo de aprendizagem, 
especificamente a utilização de técnicas de jogos (games), conhecida como gamificação (gamefication). Portanto, o armazenamento do caminho pelo conteúdo (conceitos) ao longo do processo de aprendizagem dos estudantes utilizando técnicas de gamificação e a vinculação desse conteúdo aos processos de avaliação realizados pelo sistema SAA podem garantir uma maior efetividade no processo de ensino-aprendizagem das disciplinas.

\section{Objetivo geral do projeto}

Este projeto tem como objetivo desenvolver um módulo de gestão de conteúdo (conceitos) baseado em gamificação e atrelado ao Sistema de Avaliação da Aprendizagem (SAA).

\section{Objetivos específicos}

- Estudar o funcionamento e estrutura do Sistema de Avaliação da Aprendizagem (SAA) e as estratégias de implementação de metodologias ativas e processos de avaliações formativas;

- Estudar os processos e estratégias de aplicação de técnicas de gamificação ao longo de conteúdos didáticos.

- Remodelar a estrutura de banco de dados e serviços (webservices) do Sistema de Avaliação da Aprendizagem (SAA) a fim de suportar a ligação mais forte entre conteúdos e avaliações;

- Desenvolver e testar um módulo gerenciador de conteúdo (conceitos) que suporte a implementação de técnicas de gamificação e seja atrelado ao SAA.

\section{Referencial teórico}

\subsection{O processo de Gamificação}

Os valores e benefícios educacionais dos jogos, são reconhecidos em todas as idades do Ser Humano pela sua prática, e podem ser comprovadas, a partir dos primeiros anos de vida.

Durante a infância os jogos não são meros passatempos, as brincadeiras facilitam o desenvolvimento da resistência física, da força e da coordenação motora sem falar no favorecimento de uma ótima socialização, pela atuação em várias esferas sociais que se constituem para a vida afetiva. O jogo facilita o desenvolvimento 
intelectual pelo exercício da atenção e imaginação. (PEREIRA; 1980; pg. 31)

Conforme Piaget citado por (Wadsworth, 1984, p. 44),

O jogo lúdico é formado por um conjunto linguístico que funciona dentro de um contexto social; possui um sistema de regras e se constitui de um objeto simbólico que designa também um fenômeno. Portanto, permite ao educando a identificação de um sistema de regras que permite uma estrutura sequencial que especifica a sua moralidade.

Ainda nesse ponto, Friedman (1996, p. 41) considera que:

Os jogos permitem uma situação educativa cooperativa e interacional, ou seja, quando alguém está jogando está executando regras do jogo e ao mesmo tempo, desenvolvendo ações de cooperação e interação que estimulam a convivência em grupo.

As últimas pesquisas na área de Gamificação, que se trata do uso mecânico dos jogos, com o objetivo de incrementar a participação e gerar engajamento e comprometimento por parte dos estudantes, vem apontando ganhos significativos na sua utilização.

O que se observa é que os estudantes, na sua grande maioria, querem algo diferente das aulas tradicionais. Os jogos didáticos ou a gamificação possibilitam essa mudança de comportamento em sala de aula, tornando as atividades educacionais mais atraentes aos olhos dos estudantes.

O processo de gamificação (ou transformação de conjuntos de ações em jogos didáticos) tem como objetivo proporcionar determinadas aprendizagens, diferenciandose do material pedagógico, por conter o aspecto lúdico (Cunha, 1988). Isso é utilizado para atingir determinados objetivos pedagógicos, transformando-se em uma alternativa para se melhorar o desempenho dos estudantes em alguns conteúdos de difícil aprendizagem / assimilação (Gomes et al, 2001).

Nesse ponto de vista, o jogo didático não representa o fim de um processo ou apenas um produto final, mas sim um instrumento canalizador da aprendizagem que conduz a um conteúdo didático específico. O resultado está na utilização de ações lúdicas para a aquisição de novas informações e produção de novos conhecimentos para o estudante.

Segundo pesquisas recentes, cinquenta por cento dos jovens da Geração Y (entre 13 e 33 anos) afirmam que aplicam a mecânica dos jogos em, pelo menos, um aspecto de seus cotidianos. Este grupo representa um quarto da população mundial. ${ }^{[1]}$ Corroborando com essa constatação, o processo de gamificação (no contexto mundial) tem sido uma das áreas da Tecnologia da Informação que mais tem crescido nos últimos anos. Em 
2013 movimentou US $\$ 450$ milhões e a estimativa é que em 2018 chegue próximo aos US $\$ 5,5$ bilhões. $^{[2]}$

A Figura 2 a seguir, ilustra alguns aspectos de melhoria com a utilização da gamificação. No caso específico da aprendizagem, está atrelada a descoberta de conteúdo, a qual recebe um significativo aumento em torno de $68 \%$.

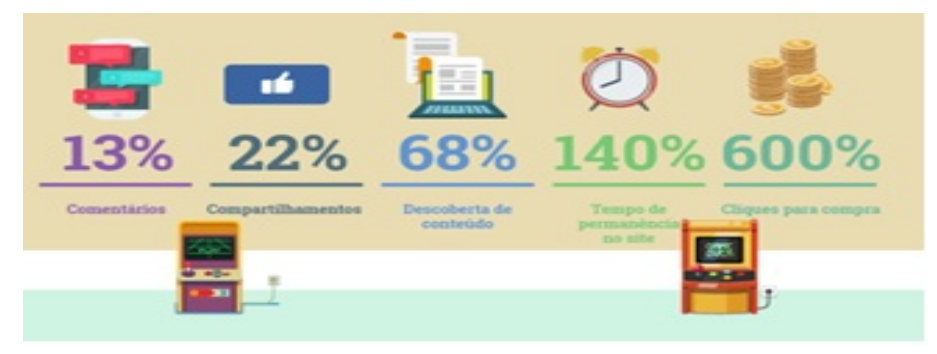

Figura 2: Alguns aspectos de melhoria com a utilização de processos de gamificação (Fonte: https://viverdeblog.com/gamificacao/)

Assim, os jogos didáticos podem ser considerados como uma importante alternativa educacional por proporcionarem o desenvolvimento das áreas afetiva, cognitiva, social, linguística, motora e moral, e por contribuírem para fomentar a criatividade, autonomia, responsabilidade, criticidade e cooperação dos envolvidos (CAMPOS; BORTOLOTO; FELÍCIO, 2003).

As principais técnicas de gamificação utilizadas ao longo dos processos educacionais são os distintivos, os níveis, as recompensas, os avatares, os desafios, os quadros de liderança, a surpresa e a cooperação.

\subsection{O fortalecimento da ligação Conteúdo-Avaliação}

De uma forma geral, os conteúdos disponibilizados nos cursos superiores de graduação, seja na modalidade presencial ou a distância, são apresentados em um formato estático. Geralmente, esses conteúdos são divididos em módulos (Mn), com avaliação (Avn) formal no final de cada um desses módulos, caracterizada como Avaliação Somativa[3] ou tradicional (Avsn). A Figura 3, ilustra este esquema geral.

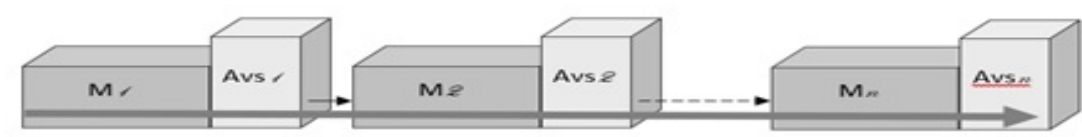

Figura 3: Esquema genérico de apresentação de conteúdos em cursos Online. 
Da forma apresentada na Figura 3, fica claro que a verificação da aprendizagem durante o processo não existe de maneira formal, sendo realizada por alguns docentes de maneira informal. Por essa razão, uma melhor estruturação dos cursos deve ser buscada. Uma das possibilidades é a utilização da proposta por Piva Jr. (PIVA JR., 2005), ilustrada na Figura 4.

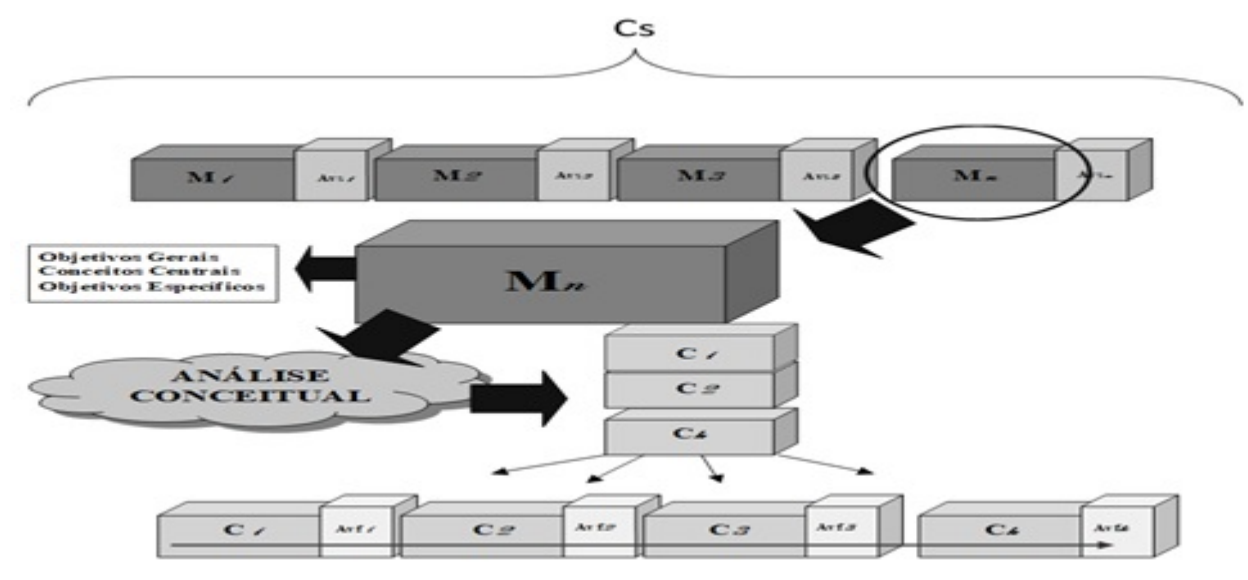

Figura 4: Planejamento de um Módulo de um curso Online (PIVA JR., 2005).

Como pode ser observado, nessa proposta, um curso (Cs) seria composto por um conjunto finito de Módulos $(\mathrm{Mn})$ e atreladas a esses módulos estariam as Avaliações Somativas (Avsn). A sua representação consiste em:Cs $=\{(M 1, A v s 1),(M 2, A v s 2),(M 3$, Avs3), ..., (Mn, Avsn)\}

Conforme ilustrado na Figura 4, ao isolar e analisar um módulo (Mn) específico de um curso (Cs), conclui-se que tal módulo tem um objetivo geral e para atingi-lo, ao longo do curso, deve ser trabalhado uma gama de conceitos $(\mathrm{C} 1, \mathrm{C} 2, \ldots, \mathrm{Ck})$ inter-relacionados, cada qual com seus objetivos específicos.

Dessa forma, ao correlacionar as Aulas em seus respectivos Conceitos (Ck) e aplicar os processos avaliativos em maior escala (ao final de cada aula), consegue-se, por meio de ferramentas computacionais adequadas, identificar e armazenar informações comportamentais dos estudantes perante os conteúdos e sua aprendizagem. Um modelo simplificado de arranjo de conteúdo de cada Aula é apresentado na Figura 5. Nela é ilustrada uma visão sintética de como o núcleo de inferências do sistema SAA conduz o processo de avaliação em torno dos conceitos estudados. Como pode ser identificado, um Conceito (Ck) é composto por três partes básicas: Proposta Pedagógica (PPk); Conteúdos Pedagógicos e Mídias (CPk) e Questões para Verificação do Aprendizado (Qk). 
Todos esses conteúdos devem ser armazenados em Base de Dados, o que facilita a sua manutenção e posterior e constante recuperação. Essa forma de construção, edição e armazenamento do curso neste formato foi definida por Piva Jr. (PIVA JR., 2005) para a adoção de cursos online (facilmente adaptado a outras modalidades) e utilizando diversos níveis de conteúdo. Para este projeto específico, vamos utilizar apenas um nível de conteúdo e a adoção de um número variável de questões cadastradas, com sua recuperação realizada randomicamente. Além disso, a junção entre conteúdo e avaliação deverá ser fortalecida pelo armazenamento do caminho percorrido ao longo do conteúdo pelos estudantes, utilizando técnicas de gamificação. Portanto, a estrutura ilustrada na Figura 5 é dividida em duas partes. A que pertence ao escopo do sistema SAA e aquela que será objeto de estudo e implementação do presente projeto: novo gerenciador de conteúdo.

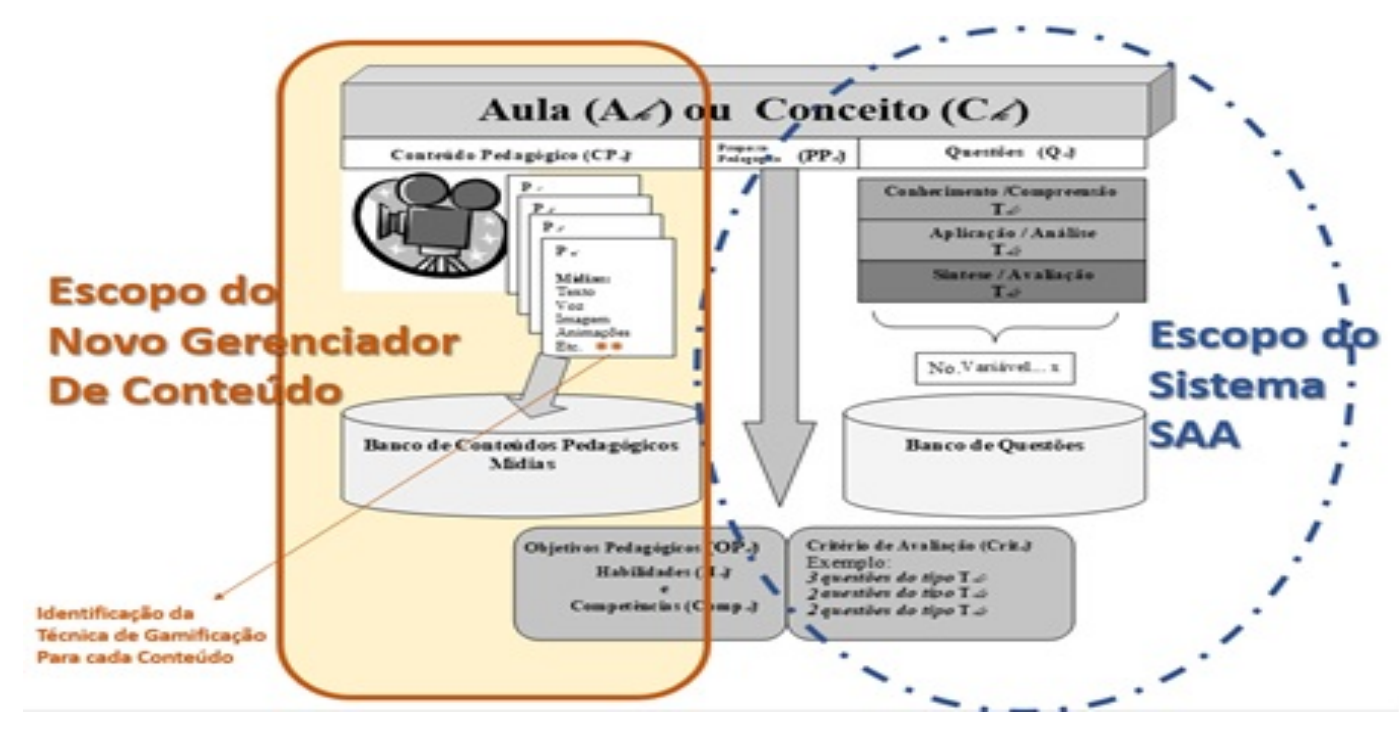

Figura 5: Arranjo dos conteúdos pedagógicos e questões de cada Aula/Conceito, separados por escopo dos sistemas (SAA e Gerenciador de Conteúdos) e a implementação de técnicas de gamificação.

\section{Metodologia de desenvolvimento}

Para atingir os objetivos específicos propostos e, por sua vez, o objetivo geral deste projeto, deve-se, primeiramente, se concentrar nos estudos sobre os processos de gamificação e suas as técnicas para utilização ao longo de conteúdos educacionais. Uma vez mapeada a estrutura dos conteúdos pedagógicos para representação da gamificação, será o momento de se concentrar no sistema SAA, entendendo o seu funcionamento, e depois, suas estruturas internas, principalmente os processos e o banco de dados. Só então, haverá condições de propor e implementar as alterações necessárias nas estruturas internas do SAA, para prepará-lo e adequá-lo para suportar a 
ligação com o novo módulo do sistema de gestão de conteúdos e gamificação. Depois de estruturado o sistema, deve-se partir para o desenvolvimento do módulo gestor de conteúdo. Ao seu final, deve testá-lo, em casos reais. Por fim, extrair os resultados e construir o relatório científico final.

\section{Resultados esperados}

Ao final do período de doze meses, espera-se que o módulo de gerenciamento de conteúdo e processos de gamificação esteja concluído e devidamente acoplado ao SAA.

\section{Considerações Finais}

Acredita-se que este projeto será parte importante da pesquisa em desenvolvimento sobre a implementação de metodologias ativas em cursos superiores de tecnologia, possibilitando uma melhor utilização do sistema SAA, o que poderá resultar em melhorias no processo de aprendizagem e, como consequência, uma possível redução nos índices de evasão dos cursos que utilizarem a ferramenta.

\section{Referências}

CAMPOS, L. M. L.; BORTOLOTTO, T. M.; FELICIO, A. K. C. A Produção de jogos didáticos para o ensino de Ciências e Biologia: uma proposta para favorecer a aprendizagem. São Paulo: UNESP, 2003. Disponível em:

CUNHA, N. Brinquedo, desafio e descoberta. Rio de Janeiro: FAE. 1988.

FRIEDMANN, Adriana. Brincar, crescer e aprender: o resgate do jogo infantil. São Paulo: Moderna, 1996.

GOMES, R. R.; FRIEDRICH, M. A Contribuição dos jogos didáticos na aprendizagem de conteúdos de Ciências e Biologia. In: EREBIO,1, Rio de Janeiro, 2001.

KISHIMOTO, T. M. Jogo, brinquedo, brincadeira e a educação. Cortez, São Paulo, 1996.

MORAES, D.A.F. (2014). A prova formativa na educação superior: possibilidade de regulação e autorregulação. Estudos de Avaliação Educacional, 25(58), p. 272-294. 
PEREIRA, Lamartine. Biblioteca Educação é Cultura: Esportes. Rio de Janeiro: Bloch; Brasília: Ministério da Educação e Cultura, 1980.

PIVA JR., Dilermando. AUXILIAR-CONSTRUTOR: uma ferramenta computacional que potencializa a ação docente no desenvolvimento de módulos de ensino de engenharia em cursos online. Dissertação de Mestrado. Orientador: Mauro Sérgio Miskulin. Campinas, SP: FEEC-UNICAMP, 2005.

PIVA JR., D.; CORTELAZZO, A.L.; FREITAS, F.A. e BELO, R.O. Sistema de Avaliação da Aprendizagem (SAA): Operacionalização da Metodologia Flipped Classroom. In: Anais do 22 Congresso Internacional ABED Educação a Distância, Águas de Lindóia, SP: ABED, 2016.

WADSWORTH, Barry. Jean Piaget para o professor da pré-escola e $1^{\circ}$ grau. São Paulo, Pioneira, 1984.

[1] Informação disponibilizada no Infográfico "O que é gamificação", disponível no endereço < http://www.mjv.com.br/biblioteca/infografico-o-que-e-gamificacao/ > acessado em $<14 / 03 / 2018>$.

[2] Pesquisa da M2 Intelligence, disponibilizado no endereço acessado em $<14 / 03 / 2018>$.

[3] Avaliação Somativa: ocorre ao final da instrução com a finalidade de verificar o que o aluno efetivamente aprendeu; inclui conteúdos mais relevantes e os objetivos mais amplos do período de instrução; visa à atribuição de notas; fornece feedback ao aluno (informa-o quanto ao nível de aprendizagem alcançado), se este for o objetivo central da avaliação formativa; presta-se à comparação de resultados obtidos com diferentes alunos, métodos e materiais de ensino. (BLOOM et al, 1976). 